\title{
Neutrophilic inflammation in stroke
}

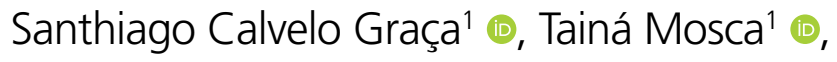 \\ Rubens José Gagliardi² ${ }^{\oplus}$, Wilma Carvalho Neves Forte ${ }^{1 *}$ (a)
}

\section{INTRODUCTION}

Stroke is one of the main diseases affecting society, i.e., in Brazil, it is the second leading cause of death and the first in the case of disabilities. From a neuroanatomical perspective, it can manifest itself in two ways, namely, hemorrhagic and ischemic $^{1}$. In both cases, neuronal tissue can be damaged, which results in the lysis of tissue cells ${ }^{1}$. As a result of this lysis, some substances are released in the injured territory, thereby initiating a local inflammatory process ${ }^{2-4}$.

Inflammation is the process by which the immune system tries to eliminate an offending agent by means of different mechanisms, in an attempt to restore the body's homeostasis 5 .

The neuroinflammation in stroke occurs due to bleeding or ischemia. Neurons and glial cells suffer from the effects of ischemia and exhibit lysis, thus releasing pathogen-associated molecular patterns (PAMPs) and damage-associated molecular patterns (DAMPs), which in turn activate microglia ${ }^{2-4}$. Microglia release cytokines and chemokines, attracting inflammatory cells to the central nervous system $(\mathrm{CNS})^{2}$. Different cells infiltrate into the CNS within a few hours, increasing brain damage and thus worsening the prognosis ${ }^{2,3}$.

\section{OBJECTIVE}

This study aims to review the neutrophilic inflammatory in stroke.

\section{METHODS}

A narrative review of the literature over the last 20 years (20002020) was carried out. The review was based on the following descriptors: stroke, inflammation, CNS, neutrophils, microglia, and cytokines. Inclusion criterion is as follows: only articles published in Portuguese or English were considered. The following bibliographic databases/search engines were used: LILACS, SciELO, and PubMed.

\section{RESULTS}

\section{Neutrophils in Stroke}

It became evident that the presence of a large number of neutrophils is related to more severe strokes, with worse clinical progression $^{2}$.

Neutrophils are the first cells of the immune system to reach the CNS following a stroke ${ }^{2}$. Their peak concentration is reached, between the first and third days after the stroke, modifying the injured environment ${ }^{4}$. This significant increase is due to the release of cytokines by local cells, such as the microglia that have become activated shortly after injury was sustained. Neutrophils reach the CNS in two ways as follows: passively, after the loss of vessel integrity in the ischemic core or through the leptomeninges; or actively, by means of the transendothelial migration of post-capillary venules reaching to the perivascular spaces following ischemia ${ }^{4}$.

After neutrophil migration, these cells become activated. They start to release proteases, elastases, reactive oxygen species (ROSs), and neutrophil extracellular traps (NETs) ${ }^{2}$. Such mechanisms, as well as the release of more cytokines by the cells at the site, accelerate the inflammatory process, aggravating the ischemic process and causing additional damage ${ }^{2}$.

NETs, in addition to their role in the attempt to eliminate pathogens, can activate the coagulation cascade, which in turn generates thrombi and worsens ischemia. In contrast, platelets also lead to the release of NETs when they come into contact with neutrophils, through the action of platelet P-selectin, thus generating a self-activation cycle ${ }^{6}$.

\footnotetext{
'Santa Casa de São Paulo, Faculdade de Ciências Médicas, Disciplina de Imunologia - São Paulo (SP), Brazil.

2Irmandade da Santa Casa de Misericórdia de São Paulo, Disciplina de Neurologia - São Paulo (SP), Brazil.

*Corresponding author: wilmanevesforte@yahoo.com.br

Conflicts of interest: the authors declare there are no conflicts of interest. Funding: none.

Received on March 25, 2021. Accepted on May 23, 2021.
} 
Among neutrophil-released proteases, metalloproteinases (MMP) stand out since they can degrade extracellular components such as laminae, fibronectin, and type IV collagen. Metalloproteinase-9 (MMP-9), in addition to degrading these components, can lead to injuries to the blood-brain barrier, rendering brain microvilli fragile and predisposing them to rupture and hemorrhage.

The elastase released by neutrophils acts in a similar way to that of MMPs. Neutrophil elastase is released during inflammation and has some paracrine effects as follows: it acts on the platelet activating factor; increases the release of CXCL-8 by local endothelial cells; degrades structural matrix proteins, such as elastin, collagens, and fibronectin, resulting in increased vascular permeability and hemorrhage in non-brain tissues; degrades additional substrates of the extracellular matrix, interrupting the homeostasis of neurons and glial cells; activates clotting during systemic infections; mediates the expression of the intracellular adhesion molecule-1 (ICAM-1) in CD11+/CD18+ endothelial cells and neutrophils; and regulates the transendothelial migration of leukocytes into peripheral venules in response to proinflammatory stimuli. Inhibition and genetic exclusion of neutrophilic elastase result in less edema, interruption of damage to the blood-brain barrier, and reduced leukocyte adhesion to cortical venule endothelial cells, resulting in reduced infarction volume ${ }^{7}$.

\section{Neutrophil Phenotypes in Stroke}

In murine models of sepsis ${ }^{8}$ and cancer ${ }^{9}$, the existence of neutrophils with proinflammatory and anti-inflammatory characteristics, called N1 and N2, respectively, was demonstrated. $\mathrm{N} 1$ neutrophils have been described as CD11 $\mathrm{b}^{\text {low }}$ cells, which produce IL-12 and exhibit cytotoxic activity against tumor cells, whereas N2 neutrophils, CD1 $1 b^{\text {high }}$, produce IL-10, ROS and have the ability to inhibit TCD8 ${ }^{+}$lymphocyte activation. More recent studies have shown that N1 and N2 neutrophils can be found in individuals with cancer ${ }^{10}$, severe inflammation $^{11}$ or vascular diseases ${ }^{12}$.

$\mathrm{N} 2$ neutrophils can also be characterized by the expression of arginase I (ArgI), CCL2, CCL5, Ym1, and mannose receptor $(\mathrm{CD} 206)^{13}$. ArgI leads to L-Arg depletion, thereby suppressing the functions of $T$ lymphocytes, since $T$ cells fail to express the CD3 subunit, necessary for their transduction and proliferation, thus causing immunosuppression ${ }^{14}$. Ym1, in turn, is associated with matrix reorganization, wound healing, negative regulation of inflammation; the expression thereof in brain has been associated with neuroprotection. The expression and secretion of $Y \mathrm{~m} 1$ by neutrophils is important in neuroprotection after cerebral ischemia. Finally, CD206 serves as a marker antigen for the neuroprotective phenotype ${ }^{13}$.
N2 neutrophils have some peculiarities, such as the presence of the Peroxisome Proliferator-Activated Receptor-gamma (PPAR- $\gamma)$ receptor, which is associated with neuroprotection and inflammation resolution after stroke, since it induces neutrophil polarization toward the N2 phenotype. The PPAR- $\gamma$ receptor also decreases microglia/macrophage activation markers and neutrophil infiltration in the brain, two or three days after stroke onset ${ }^{3}$. In addition, activation of this receptor leads to an earlier infiltration of neutrophils, as well as dispersion of this cell type after about two days. The N2 phenotype promotes its own clearance by means of phagocytosis, since the activation of its receptor increases neutrophil clearance. N2 phagocytosis results in the release of anti-inflammatory mediators, contributing to create a favorable scenario for the brain's recovery process following stroke. However, the attempt so far to treat patients with the PPAR- $\gamma$ agonist has failed to induce neuroprotection by neutrophil depletion ${ }^{13}$.

Other important receptors involved in the recognition of neutrophil phenotypes are TLRs ${ }^{15}$. Such receptors, in conjunction with others, are responsible for discriminating among various pathogen recognition patterns. They are present in different cells of the immune system, such as neutrophils, macrophages, and monocytes ${ }^{16}$. In neutrophils, TLR 4 is important since it also plays a role in neutrophils polarization and function. The absence of TLR 4 increases the infiltration of neutrophils and induces a polarization of these cells toward the N2 phenotype. In general, TLR 4 inhibition or blockade reduces inflammation and ischemic damage, preventing hemorrhagic transformation induced by tissue plasminogen activator ( $\mathrm{t}-\mathrm{PA})$, a possible treatment for stroke ${ }^{2}$, stimulating neutrophils toward cytoprotective phenotypes ${ }^{15}$.

\section{Microglia and Neutrophilic Inflammation in Stroke}

Microglia, activated by DAMPs and PAMPs, release cytokines and chemokines that attract immune cells to the affected site ${ }^{2}$. In addition to this attractive effect, microglia have the function of controlling the concentration of neutrophilic infiltrate in the area injured by the stroke. This control is maintained by phagocytosis, which is in turn performed by neutrophils that are in excess. Thus, microglia remove neutrophils from the parenchyma, perivascular, and subpial spaces following brain ischemia ${ }^{4}$.

At the periphery of the infarction, microglia continue to proliferate and increase in number. In this way, these cells phagocyte neutrophils present at the periphery of the injury, preventing the accumulation thereof in that region. Neutrophils in the core of the lesion do not undergo clearance, which results in their accumulation in the core of the stroke injury. For these 
reasons, microglial dysfunction and lysis are associated with the accumulation of neutrophils in injured brain tissue ${ }^{4}$, worsening the prognosis of stroke patients. The microglia recognize both endothelial damage and neutrophilic invasion, and in a cooperative fashion, microglia and the blood-brain barrier form cytoplasmic processes that physically protect activated endothelia and capture infiltrated neutrophils ${ }^{17}$.

\section{Mast cells and Neutrophilic Inflammation in Stroke}

Mast cells represent the first line of defense against toxic agents and brain damage. They contain granules with potent preformed substances as follows: vasoactive (histamine and bradykinin), anticoagulant (heparin), proteolytic (tryptase and chymase), and chemotactic factors, which are released upon their activation. This activation has two phases as follows: secretion of preformed granules and de novo synthesis of new granules having vasoactive mediators, chemotactic factors (prostaglandins, platelet activation factor, and leukotrienes), and cytokines (tumor necrosis factor [TNF] $)^{18}$.

Mast cells release chemotactic factors, platelet-activating factor, TNF, IL-4, IL-5, and CXCL- $8{ }^{18}$. These substances attract neutrophils, macrophages, and eosinophils.

Mast cells also participate in the regulation of early postischemic deficiency, brain swelling, and inflammation, in addition to MMP-2 and MMP-9, which degrade type IV collagen. The constituents of mast cell granules, especially histamine, heparin, and bradykinin, produce microcirculatory effects, and the modulation of their release during ischemia influences the extent of ischemic injury ${ }^{19}$.

\section{Interleukins 1, 6, and 10 in Stroke}

As in other inflammatory processes, several cytokines are involved in stroke and, in particular, IL-1. This cytokine is an important mediator of acute brain injury and regulates inflammation during the host's defense response. IL-1 also induces the expression of a series of mediators, several of which form part of the systemic inflammatory response after stroke, especially acute phase proteins ${ }^{15}$. Thus, IL-1 increases ischemic damage to an extent that is similar to that of lipopolysaccharide, in addition to exacerbating cerebral edema and worsening stroke ${ }^{18}$.

IL-1 acts synergistically with IL- 6 and chemokines, especially CXCL-8, resulting in neutrophil mobilization and greater cortical infiltration, even before the changes that are brought about by ischemic damage. Therefore, acute systemic inflammatory stimuli by IL- 1 and IL- 6 are harmful after a stroke, with IL-1 being a critical mediator in this condition ${ }^{19}$.

Conversely, IL-10, an immunoregulatory cytokine synthesized by CNS cells, inhibits IL-1 production by decreasing the expression of IL-1 receptors and has been related to an improvement in the ischemic condition during the acute phase of stroke, as our group has previously described ${ }^{20}$.

\section{Stroke Recurrence}

Patients who had a stroke are more likely to suffer from another stroke when compared with individuals without this history ${ }^{3}$. Neutrophils are associated with this recurrence, as they interact with clotting factors and platelets, activating the coagulation cascade shortly after ischemia ${ }^{2,3}$. In addition, the intravascular accumulation of neutrophils prevents the local blood flow, thereby generating a new ischemia ${ }^{21}$.

A factor that increases the chance of recurrence is systemic infections, either acute or chronic ones, as they are triggers for the occurrence of stroke and are associated with a less favorable clinical outcome. Infections modulate the incidence of stroke and the functional recovery of the affected area. Systemic inflammatory stimuli act in conjunction with the ischemic condition, amplifying innate systemic immune responses and leading to exacerbated brain damage. Hence, the systemic inflammatory pathways provide a point of convergence and synergism when the stroke coincides with an associated inflammatory stimulus ${ }^{19}$.

\section{Inflammatory Markers Associated with the Prognosis of Stroke Patients}

Cerebral edema is indicative of a poor stroke prognosis and affects two thirds of stroke patients ${ }^{12}$. The greater number of neutrophils is related to greater severity, size of ischemic infarction, greater area of cerebral infarction, a tendency to hemorrhage in stroke. In addition, they can bring about a second ischemic wave, further worsening tissue damage caused by the first wave, which is predictive of a worse prognosis. A high neutrophil count at hospital admission is associated with an increased risk of a new stroke in a high-risk population. Death, about three months after stroke, may be associated with the interaction among endothelium, platelets, and NETs hyperactivity.

Another relevant marker is the neutrophil-lymphocyte ratio, a marker for an easy detection of systemic inflammation. A high neutrophil-lymphocyte ratio is associated with a worse prognosis and predicts mortality within a short period of time. The neutrophil-lymphocyte ratio is correlated with the infarction volume in the anterior circulation stroke but not with that in the posterior circulation stroke $\mathrm{e}^{22}$.

Some cytokines can assist in the prognosis of stroke patients' progression. The large increase in cytokines indicates a poor prognosis, since they are associated with the maintenance of inflammation and attraction of cells, such as neutrophils, to the ischemic region ${ }^{18}$. IL-6, as well as C-reactive protein, is probably ominous inflammation markers as far as stroke 
Chart 1. Inflammatory components in stroke.

\begin{tabular}{|l|r|}
\hline Components & Large number is related to more severe strokes and worse clinical progression. \\
\hline Neutrophils & $\begin{array}{r}\text { Dysfunction and lysis are associated with accumulation of neutrophils in central nervous system. } \\
\hline \text { Mast cells }\end{array}$ \\
\hline Cytokines & $\begin{array}{r}\text { Participate in the regulation of post-ischemic deficiency, brain swelling and inflammation. } \\
\text { process; interleukin-10: decreases the inflammatory process. }\end{array}$ \\
\hline
\end{tabular}

progression is concerned ${ }^{21}$. Conversely, high levels of IL-10 were correlated with a better prognosis in the acute phase of stroke ${ }^{20}$. The components involved in stroke and discussed in this article are shown in Chart 1.

\section{CONCLUSION}

Having the knowledge about the neutrophilic inflammatory process that occurs in stroke has attracted increasing interest, especially as it is a means of decreasing and improving prognosis. In the near future, it is even anticipated to allow for stroke prevention.

\section{AUTHORS' CONTRIBUTIONS} SCG, TM, RJG, WCNF: Conceptualization, Data curation, Formal Analysis, Writing - original draft, Writing - review $\&$ editing. All authors contributed equally to this study.

\section{REFERENCES}

1. Hankey GJ. Stroke. Lancet. 2016;13:1-10. https://doi. org/10.1016/S0140-6736(16)30962-X

2. Liu H, Wang R, Shi J, Zhang Y, Huang Z, You S, et al. Baseline neutrophil counts and neutrophil ratio may predict a poor clinical outcome in minor stroke patients with intravenous thrombolysis. J Stroke Cerebrovasc Dis. 2019;28(11):104340. https://doi.org/10.1016/j.jstrokecerebrovasdis.2019.104340

3. Zhu B, Liu H, Pan Y, Jing J, Li H, Zhao X, et al. Elevated neutrophil and presence of intracranial artery stenosis increases the risk of recurrent stroke. Stroke. 2018;49(10):2294-300. https:// doi.org/10.1161/STROKEAHA.118.022126

4. Otxoa-de-Amezaga A, Miró-Mur F, Pedragosa J, Gallizioli M, Justicia C, Gaja-Capdevila N, et al. Microglial cell loss after ischemic stroke favors brain neutrophil accumulation. Acta Neuropathol. 2019;137(2):321-41. https://doi.org/10.1007/ s00401-018-1954-4

5. Forte WCN. Defesa imunológica contra agentes infecciosos. In: Forte WCN. Imunologia: do básico ao aplicado. 3th ed. São Paulo: Editora Atheneu; 2015. p. 275-85.

6. Valles J, Lago A, Santos MT, Latorre AM, TembI JI, Salom JB, et al. Neutrophil extracellular traps are increased in patients with acute ischemic stroke: prognostic significance. Thromb Haemost. 2017;117(10):1919-29. https://doi.org/10.1160/ TH17-02-0130

7. Stowe AM, Adair-Kirk TL, Gonzales ER, Perez RS, Shah AR, Park TS, Gidday JM. Neutrophil elastase and neurovascular injury following focal stroke and reperfusion. Neurobiol Dis. 2009;35(1):82-90. https://doi.org/10.1016/j.nbd.2009.04.006
8. Tsuda Y, Takahashi H, Kobayashi M, Hanafusa T, Herndon DN, Suzuki F. Three different neutrophil subsets exhibited in mice with different susceptibilities to infection by methicillin-resistant Staphylococcus aureus. Immunity. 2004;21(2):215-26. https:// doi.org/10.1016/j.immuni.2004.07.006

9. Fridlender ZG, Sun J, Kim S, Kapoor V, Cheng G, Ling L, Worthen GS, Albelda SM. Polarization of tumor-associated neutrophil phenotype by TGF-beta: "N1" versus "N2" TAN. Cancer Cell. 2009;16(3):183-94. https://doi.org/10.1016/j. ccr.2009.06.017

10. Acharyya S, Massague J. Arresting supporters: targeting neutrophils in metastasis. Cell Res. 2016;26(3):273-4. https:// doi.org/10.1038/cr.2016.17

11. Pillay J, Kamp VM, Hoffen E, Visser T, Tak T, Lammers $J-W$, et al. A subset of neutrophils in human systemic inflammation inhibits T cell responses through Mac-1. J Clin Invest. 2012;122(1):327-36. https://doi.org/10.1172/ JCI57990

12. Andrade JBC, Mohr JP, Lima FO, Barros LCM, Nepomuceno $C R$, Portela LB. Predictors of hemorrhagic transformation after acute ischemic stroke based on the experts' opinion. Arq Neuropsiquiatr. 2020,78(7):390-6. https://doi. org/10.1590/0004-282×20200008

13. Cuartero MI, Ballesteros I, Moraga A, Nombela F, Vivancos J, Hamilton JA, et al. N2 neutrophils, novel players in brain inflammation after stroke: modulation by the PPAR $\gamma$ agonist rosiglitazone. Stroke. 2013;44(12):3498-508. https://doi. org/10.1161/STROKEAHA.113.002470 
14. Sippel TR, Shimizu T, Strnad F, Traystman RJ, Herson PS, Waziri A. Arginase I release from activated neutrophils induces peripheral immunosuppression in a murine model of stroke. J Cerebr Blood F Met. 2015;35(10): 1657-63. https://doi. org/10.1038/jcbfm.2015.103

15. García-Culebras A, Durán-Laforet $\vee$, Peña-Martínez C, Moraga A, Ballesteros I, Cuartero MI, et al. Role of TLR4 (Toll-Like Receptor 4) in N1/N2 neutrophil programming after stroke. Stroke. 2019;50(10):2922-32. https://doi.org/10.1161/ STROKEAHA. 119.02508

16. Kawai T, Akira S. TLR signaling. Semin Immunol. 2007;19(1):2432. https://doi.org/10.1016/j.smim.2006.12.004

17. Neumann J, Riek-Burchardt M, Herz J, Doeppner TR, König R, Hütten $\mathrm{H}$, et al. Very-late-antigen-4 (VLA-4)-mediated brain invasion by neutrophils leads to interactions with microglia, increased ischemic injury and impaired behavior in experimental stroke. Acta Neuropathol. 2015;127(2):259-77. https://doi. org/10.1007/s00401-014-1355-2

18. Strbian D, Karjalainen-Lindsberg M-J, Tatlisumak T, Lindsberg PJ. Cerebral mast cells regulate early ischemic brain swelling and neutrophil accumulation. J Cerebr
Blood F Met. 2005;26(5):605-12. https://doi.org/10.1038/ sj.jcbfm. 9600228

19. McColl BW, Rothwell NJ, Allan SM. Systemic inflammatory stimulus potentiates the acute phase and CXC chemokine responses to experimental stroke and exacerbates brain damage via interleukin-1- and neutrophil-dependent mechanisms. J Neurosci. 2007;27(16):4403-12. https://doi.org/10.1523/ JNEUROSCI.5376-06.2007

20. Protti G, Gagliardi RJ, Forte WCN. Interleukin-10 may protect against progressing injury during the acute phase of ischemic stroke. Arq Neuropsiquiatr. 2013;71(11):846-51. https://doi. org/10.1590/0004-282X20130168

21. Zhu B, Pan $Y$, Jing J, Meng $X$, Zhao X, Liu L, et al. Neutrophil counts, neutrophil ratio, and new stroke in minor ischemic stroke or TIA. Neurology. 2018;90(21):1870-8. https://doi. org/10.1212/WNL.0000000000005554

22. Kocaturk O, Besli F, Gungoren F, Kocaturk M, Tanriverdi Z. The relationship among neutrophil to lymphocyte ratio, stroke territory, and 3-month mortality in patients with acute ischemic stroke. Neurol Sci. 2018;40(1):139-46. https://doi. org/10.1007/s10072-018-3604-y 